\title{
The Shannon and Rényi Differential Entropies of the Weighted Sum of Squared Nakagami- $m$ Random Variables
}

\author{
Antonio Alisson Pessoa Guimarães and Charles Casimiro Cavalcante
}

\begin{abstract}
This paper investigates the Shannon and Rényi differential entropies over the weighted sum of squared Nakagami- $m$ mutually independent random variables. Based on the probability density function (PDF) of the sum of Gamma variables, we provide, in terms of special functions, closed-form expressions for such entropy measures. Finally, we present the numerical results of a particular case involving the weighted sum of two squared Nakagami- $m$ random variables, in order to graphically represent the theoretical analysis.
\end{abstract}

Keywords-Shannon differential entropy, Rényi differential entropy, Nakagami- $m$ random variable.

\section{INTRODUCTION}

In general terms, the Shannon entropy or, simply, entropy of a random variable is defined in terms of its probability distribution that quantifies the amount of information in such variable. In other words, this concept is a metric for information and has a fundamental role in Information Theory, in which is sometimes seen as a measure of randomness or uncertainty [1], [2].

In recent years, Information Theory has found many applications and, consequently, the use of this measure can be extended to other areas of knowledge, such as: communication systems [3], signal processing [4], pattern recognition [5], data mining [6], machine learning [7], thermodynamics [8], economy [9], quantum mechanics [10], among others.

Naturally, the Shannon entropy concept was widely adapted for the purpose of modeling problems of similar characteristics and, as consequence, variations and generalizations were proposed. For instance, Rényi entropy [11], Tsallis entropy [12], Sharma-Mittal entropy, it which contains Shannon, Rényi and Tsallis entropies as special cases [13].

In parallel, our particular interest in such metrics over the sum of squared Nakagami- $m$ random variables is due to the significant number of recent publications over Nakagami- $m$ fading channels in wireless communication systems. In this sense, the Nakagami- $m$ fading covers a wide range of multipath fading channels by varying the fading parameter $m$ as the one-side Gaussian, Rayleigh and Rice fading channels [14]. Furthermore, in the context of performance analysis investigation on diversity combining, diversity reception, ergodic

Antonio Alisson Pessoa Guimarães, Institute of Engineering and Sustainable Development (IEDS), University of International Integration of the Afro-Brazilian Lusophony (UNILAB), Redenção-Ceará, Brazil, e-mail alisson@unilab.edu.br; Charles Casimiro Cavalcante, Wireless Telecommunication Research Group (GTEL), Federal University of Ceará (UFC), FortalezaCeará, Brazil, e-mail: charles@gtel.ufc.br. capacity or mutual information problems, this model provides good analytical tractability in various situations [15], [16].

The use of multiple antennas at the transmitter and receiver in wireless communication systems, known as multiple-input multiple-output (MIMO) systems, into ergodic capacity problems, for example, may require the determination of the statistics over the sum of squared envelopes of Nakagami- $m$ faded signals, whose problems are equivalents to evaluate the statistics of the sum of Gamma random variables [16], [17], [18].

In turn, the entropy measures can be applied over multipath fading channels in several contexts. In [3], bounds on mutual information of Rayleigh fading channels with Gaussian input was derived, while [19] investigates the performance of energy detection-based spectrum sensing over $\mathcal{F}$ composite fading channels. Moreover, [20] provides a communication scheme for MIMO fading channels that estimates the fading via transmission of pilot symbols at regular intervals and feeds the fading estimates to the nearest neighbor decoder. More recently, [21] addresses the performance of wireless fading channels, namely, Rayleigh, Rician, and Nakagami- $m$ fading channels, taking both noiseless and additive noisy cases.

Specifically, this work provides closed-form expressions for Shannon and Rényi differential entropies considering the weighted sum of squared Nakagami- $m$ mutually independent random variables, where two situations are observed: identically and non-identically distributed Nakagami- $m$ random variables. In addition, the Shannon information will be obtained as a particular measure of the Rényi entropy [2].

In other words, we present new results for Shannon and Rényi differential entropies with simple analytical representations. Therefore, we expect that the proposed results may contribute to the investigation of performance metrics over Nakagami- $m$ fading channels and with their respective variants.

The rest of this paper is organized as follows. The next section presents important results on Nakagami- $m$ and Gamma random variables as well as the statement that relates the Shannon and Rényi entropies. Section III applies these results to derive the formulas for Shannon and Rényi entropies. Section IV provides a numerical interpretation of the entropy results. Finally, in Section V we state the conclusions.

Throughout this paper, the probability density function (PDF) and the moment generating function (MGF) of a random variable $X$ are denoted as $p_{X}(\cdot)$ and $M_{X}(\cdot)$, respectively. Furthermore, $\mathbb{E}_{X}[\cdot]$ and $\operatorname{Var}[\cdot]$ stand for expectation and variance of $X$, respectively. 


\section{Preliminary Aspects}

In this section, we establish the notation used in this paper, followed by the mathematical framework from which will be described the definitions and lemmas. The propositions of such lemmas are not necessarily novelties, but they are relevant to understand the proposed theory and also some proofs are not commonly available in the literature.

\section{A. The Gamma and Nakagami-m distributions}

A random variable $Z$ follows a Gamma distribution with parameters $\alpha>0$ e $\beta>0$ if the PDF is given by [22]

$$
p_{Z}(z)=\frac{z^{\alpha-1} e^{-z / \beta}}{\beta^{\alpha} \Gamma(\alpha)} u(z),
$$

where $u(\cdot)$ is the unit step function and $\Gamma(\cdot)$ is the gamma function [23]. In what follows we will use the notation

$$
Z \sim \gamma(\alpha, \beta)
$$

to denote that $Z$ is Gamma distributed with parameters $\alpha$ and $\beta$ which are called as the shape and scale, respectively, of the random variable. Furthermore, the MGF of $Z$ is expressed as

$$
M_{Z}(t)=\mathbb{E}_{Z}\left[e^{t Z}\right]=\left(\frac{\beta}{\beta-t}\right)^{\alpha},
$$

where $\mathbb{E}_{Z}[\cdot]$ represents the expectation (mean) operator over $Z$.

In turn, a random variable $X$ following a Nakagami-m distribution is defined by [14]

$$
p_{X}(x)=\frac{2}{\Gamma(m)}\left(\frac{m}{\Omega}\right)^{m} x^{2 m-1} e^{-(m / \Omega) x^{2}} u(x)
$$

where $m=\frac{\mathbb{E}^{2}\left[X^{2}\right]}{\operatorname{Var}\left[X^{2}\right]}$ and $\Omega=\mathbb{E}_{X}\left[X^{2}\right]$, and we represent that $X$ is Nakagami- $m$ distributed with parameters $m$ and $\Omega$ as

$$
X \sim \mathcal{N} \mathcal{K}_{m}(m, \Omega)
$$

Now, we introduce two lemmas regarding the properties of Gamma and Nakagami- $m$ random variables, which will be used in this work.

Lemma 1: If $X \sim \mathcal{N} \mathcal{K}_{m}(m, \Omega)$, then the random variable $Z=k X^{2}$, is Gamma distributed as

$$
Z \sim \gamma\left(m, \frac{k \Omega}{m}\right)
$$

where $k$ is a positive number.

Proof: The PDF of $Z$ is obtained from a simple variable transformation [22]

$$
p_{Z}(z)=\frac{1}{2 k \sqrt{z / k}}\left[p_{X}\left(\sqrt{\frac{z}{k}}\right)+p_{X}\left(-\sqrt{\frac{z}{k}}\right)\right] u(z) .
$$

Lemma 2: Let $\left\{Z_{i}\right\}_{i=1}^{n}$ be a sequence of $n$ independent Gamma random variables with parameters $\alpha_{i}$ and $\beta$, respectively. Then the random variable $X$ defined as

$$
X=\sum_{i=1}^{n} Z_{i}
$$

is Gamma distributed. Specifically, we can write that

$$
X \sim \gamma\left(\sum_{i=1}^{n} \alpha_{i}, \beta\right)
$$

Proof: In order to derive the PDF of $X$ it is sufficient to determine the associated MGF. Note that the MGF of each $Z_{i}$ is given by

$$
M_{Z_{i}}(t)=\left(\frac{\beta}{\beta-t}\right)^{\alpha_{i}}
$$

Since $\left\{Z_{i}\right\}_{i=1}^{n}$ is a sequence of $n$ independent random variables, then using this fact on the MGF of $X$ we obtain

$$
M_{X}(t)=\prod_{i=1}^{n} M_{Z_{i}}(t)=\left(\frac{\beta}{\beta-t}\right)^{\sum_{i=1}^{n} \alpha_{i}},
$$

and the proof is completed.

\section{B. The Shannon and Rényi entropies}

The Shannon differential entropy $h(Z)$ (or simply differential entropy) is a measure of randomness of a continuous random variable $Z$ in which defined as [2]

$$
h(Z)=-\int_{\mathcal{S}} p_{Z}(z) \log \left(p_{Z}(z)\right) d z,
$$

where $\mathcal{S}$ is the support set of $Z$.

In turn, the Rényi entropy $h_{r}(Z)$ of order $r>0$, with $r \neq 1$, is defined as [2]

$$
h_{r}(Z)=\frac{1}{1-r} \log \left(\int_{\mathcal{S}} p_{Z}^{r}(z) d z\right),
$$

which can be seen as a generalization of Shannon entropy [11] and the details are described as follows.

Lemma 3: Let $Z$ be a continuous random variable. The condition that relates the Shannon and Rényi entropies of $Z$ is

$$
\lim _{r \rightarrow 1} h_{r}(Z)=h(Z) .
$$

As consequence of this result and by convention it writes

$$
h_{1}(Z)=h(Z) .
$$

Proof: Firstly, we transform the analytical expressions of Shannon and Rényi entropies, respectively, in terms of the mean operator $\mathbb{E}_{Z}[\cdot]$ as

$$
h_{r}(Z)=\frac{1}{1-r} \log \left(\mathbb{E}_{Z}\left[p_{Z}^{r-1}(Z)\right]\right)
$$

and

$$
h(Z)=-\mathbb{E}_{Z}\left[\log \left(p_{Z}(Z)\right)\right] .
$$

From the fact that $\log (\cdot)$ is a continuous function, $\mathbb{E}_{Z}[\cdot]$ is a linear operator and since $p_{Z}(\cdot)$ is a positive function, we obtain

$$
\lim _{r \rightarrow 1} \log \left(\mathbb{E}_{Z}\left[p_{Z}^{r-1}(Z)\right]\right)=\log \left(\mathbb{E}_{Z}[1]\right)=\log (1)=0 .
$$

Consequently,

$$
\lim _{r \rightarrow 1} h_{r}(Z)=\frac{0}{0}
$$


whose result represents an indeterminate form. Thus, applying the L'Hôpital's Rule, we have [24]

$$
\lim _{r \rightarrow 1} h_{r}(Z)=-\lim _{r \rightarrow 1} \frac{d}{d r} \log \left(\mathbb{E}_{Z}\left[p_{Z}^{r-1}(Z)\right]\right)
$$

By convenience, we define the differentiable function $G(\cdot)$ as

$$
G(r)=\mathbb{E}_{Z}\left[p_{Z}^{r-1}(Z)\right]
$$

Then, applying the chain rule yields

$$
\frac{d}{d r} \log (G(r))=\frac{1}{G(r)} \frac{d}{d r} G(r) .
$$

Now, we need to evaluate the derivative of $G(t)$ and in this case we have

$$
\begin{aligned}
\frac{d}{d r} G(r) & =\frac{d}{d r} \mathbb{E}_{Z}\left[p_{Z}^{r-1}(Z)\right] \\
& =\mathbb{E}_{Z}\left[\frac{d}{d r} p_{Z}^{r-1}(Z)\right] \\
& =\mathbb{E}_{Z}\left[\frac{1}{p_{Z}(Z)} \frac{d}{d r} p_{Z}^{r}(Z)\right] \\
& =\mathbb{E}_{Z}\left[\frac{1}{p_{Z}(Z)} p_{Z}^{r}(Z) \log \left(p_{Z}(Z)\right)\right]
\end{aligned}
$$

where the last equality was obtained following the derivative rule [24]

$$
\frac{d}{d x} a^{x}=a^{x} \log (a), \quad a>0 .
$$

Thus, Eq. (23) reduces to

$$
\frac{d}{d r} G(r)=\mathbb{E}_{Z}\left[p_{Z}^{r-1}(Z) \log \left(p_{Z}(Z)\right)\right]
$$

and substituting such result in Eq. (22), we obtain

$$
\frac{d}{d r} \log G(r)=\frac{1}{\mathbb{E}_{Z}\left[p_{Z}^{r-1}(Z)\right]} \mathbb{E}_{Z}\left[p_{Z}^{r-1}(Z) \log \left(p_{Z}(Z)\right)\right] .
$$

Therefore, the Rényi differential entropy can be represented as

$$
h_{r}(Z)=\frac{1}{1-r} \log (G(r))
$$

and from Eq. (20) we conclude

$$
\begin{aligned}
\lim _{r \rightarrow 1} h_{r}(Z) & =-\lim _{r \rightarrow 1} \frac{d}{d r} \log (G(r)) \\
& =-\lim _{r \rightarrow 1} \frac{1}{\mathbb{E}_{Z}\left[p_{Z}^{r-1}(Z)\right]} \mathbb{E}_{Z}\left[p_{Z}^{r-1}(Z) \log \left(p_{Z}(Z)\right)\right] \\
& =-\frac{1}{\mathbb{E}_{Z}[1]} \mathbb{E}_{Z}\left[1 \cdot \log \left(p_{Z}(Z)\right)\right] \\
& =-\mathbb{E}_{Z}\left[\log \left(p_{Z}(Z)\right)\right] \\
& =h(Z),
\end{aligned}
$$

as described in Eq. (17).

\section{THE ENTROPY OF THE SUM OF SQUARED NAKAGAMI- $m$ VARIABLES}

In this section, we propose analytical expressions to Shannon and Rényi entropies of a random variable written as a weighted sum of squared Nakagami- $m$ mutually independent random variables.

Then, let $\left\{R_{i}\right\}_{i=1}^{n}$ be a sequence of $n$ mutually independent Nakagami- $m$ random variables as

$$
R_{i} \sim \mathcal{N} \mathcal{K}_{m}\left(m_{i}, \Omega_{i}\right)
$$

We also consider the convenient sequence of $n$ positive numbers $\left\{k_{i}\right\}_{i=1}^{n}$ such that

$$
k_{1} \frac{m_{1}}{\Omega_{1}}=k_{2} \frac{m_{2}}{\Omega_{2}}=\cdots=k_{n} \frac{m_{n}}{\Omega_{n}}=L,
$$

where $L>0$ is a constant. In addition, the sum of the parameters $m_{i}$, denoted by $\mu$, is greater than or equal to 1 , i.e.,

$$
\mu=\sum_{i=1}^{n} m_{i} \geq 1 .
$$

Therefore, from these assumptions, we define the continuous random variable $Z$ as

$$
Z=\sum_{i=1}^{n} k_{i} R_{i}^{2}
$$

which represents a weighted sum of squared Nakagami- $m$ variables.

In order to achieve our goals, we must initially transform $Z$ into a sum of Gamma independent random variables. Indeed, let $X_{i}$ be a random variable defined as $X_{i}=k_{i} R_{i}^{2}$ for each $i=1,2, \ldots, n$. Then, $Z$ can be written as

$$
Z=\sum_{i=1}^{n} X_{i}
$$

and, from Lemma 1, we ensure that

$$
X_{i} \sim \gamma\left(m_{i}, \frac{k_{i} \Omega_{i}}{m_{i}}\right) .
$$

According to equality restriction in Eq. (30), each variable $X_{i}$ is Gamma distributed as

$$
X_{i} \sim \gamma\left(m_{i}, L\right)
$$

Consequently, by Lemma 2, we conclude that $Z$ is also a Gamma variable with parameters $\mu=\sum_{i=1}^{n} m_{i}$ and $L$, respectively. Thus, the PDF of $Z$ is given by

$$
p_{Z}(z)=\frac{z^{\mu-1} e^{-z / L}}{L^{\mu} \Gamma(\mu)} u(z) .
$$

Now, effectively we will determine the Rényi entropy of $Z$ and in the sequence, the Shannon entropy as an special case. 


\section{A. The Rényi entropy of $Z$}

Substituting the PDF of $Z$ obtained in Eq. (36) over Rényi entropy expression (see Eq. (13)), yields

$$
h_{r}(Z)=\frac{1}{1-r} \log \left[\frac{1}{\left[L^{\mu} \Gamma(\mu)\right]^{r}} \int_{0}^{\infty} z^{\mu r-r} e^{-r z / L} d z\right],
$$

or, equivalently,

$$
h_{r}(Z)=\frac{1}{1-r} \log \left[\frac{1}{\left[L^{\mu} \Gamma(\mu)\right]^{r}} \int_{0}^{\infty} z^{(\mu r-r+1)-1} e^{-r z / L} d z\right] .
$$

The last formulation has the objective to use the following integral result [25, Eq. 3.381-4]

$$
\int_{0}^{\infty} x^{\nu-1} e^{-\omega x} d x=\frac{1}{\omega^{\nu}} \Gamma(\nu)
$$

where, $\nu>0$ and $\omega>0$. In our case, we have $\omega=r / L>0$, because $r$ and $L$ are positive numbers, and $\nu=\mu r-r+1$. The details about $\nu$ being also a positive number are described in Appendix.

Thus, from Eq. (39), we obtain

$$
\begin{aligned}
h_{r}(Z) & =\frac{1}{1-r} \log \left[\frac{1}{\left[L^{\mu} \Gamma(\mu)\right]^{r}}\left(\frac{L}{r}\right)^{\mu r-r+1} \Gamma(\mu r-r+1)\right] \\
& =\frac{1}{1-r} \log \left[\frac{r^{r-\mu r-1}}{[\Gamma(\mu)]^{r}} L^{1-r} \Gamma(\mu r-r+1)\right] .
\end{aligned}
$$

Therefore, expanding the last expression using logarithmic properties, Rényi entropy becomes

$$
\begin{aligned}
h_{r}(Z) & =\frac{1}{1-r}[(r-\mu r-1) \log (r)+(1-r) \log (L) \\
& +\log (\Gamma(\mu r-r+1))-r \log (\Gamma(\mu))],
\end{aligned}
$$

that completes the proof.

As a particular case, for independent and identically distributed (i.i.d.) Nakagami- $m$ random variables, we have $\mu=m n$ and Rényi entropy is written as

$$
\begin{aligned}
h_{r}(Z) & =\frac{1}{1-r}[(r-m n r-1) \log (r)+(1-r) \log (L) \\
& +\log (\Gamma(m n r-r+1))-r \log (\Gamma(m n))] .
\end{aligned}
$$

\section{B. The Shannon entropy of $Z$}

According to Lemma 3, Shannon entropy $h(Z)$ can be obtained from $\lim _{r \rightarrow 1} h_{r}(Z)$. Indeed, by convenience, we define the differentiable function $F(\cdot)$ as

$$
\begin{aligned}
F(r) & =(r-\mu r-1) \log (r)+(1-r) \log (L) \\
& +\log (\Gamma(\mu r-r+1))-r \log (\Gamma(\mu)) .
\end{aligned}
$$

Thus, Rényi entropy in Eq. (41) reduces to

$$
h_{r}(Z)=\frac{F(r)}{1-r} .
$$

In addition, due to the continuity of the logarithmic and Gamma functions, it follows

$$
\lim _{r \rightarrow 1} F(r)=-\mu \log (1)+\log (\Gamma(\mu))-\log (\Gamma(\mu))=0 .
$$

As consequence, it yields the indeterminate form

$$
h(Z)=\lim _{r \rightarrow 1} h_{Z}(t)=\frac{0}{0} .
$$

By L'Hôpital's Rule, we get

$$
h(Z)=-\lim _{r \rightarrow 1} \frac{d}{d r} F(r) .
$$

Note that, after some chain rules, we obtain

$$
\begin{aligned}
\frac{d}{d r} F(r) & =(1-\mu) \log (r)+\frac{r-\mu r-1}{r}-\log (L) \\
& -\log (\Gamma(\mu))+\frac{\mu-1}{\Gamma(\mu r-r+1)} \frac{d}{d t} \Gamma(t),
\end{aligned}
$$

where, $t=\mu r-r+1$. Using the fact that Digamma function is defined by [23]

$$
\psi(x)=\frac{1}{\Gamma(x)} \frac{d}{d x} \Gamma(x), \quad x \neq 0,-1,-2, \ldots,
$$

then, the derivative of $F(\cdot)$ becomes

$$
\begin{aligned}
\frac{d}{d r} F(r) & =(1-\mu) \log (r)+\frac{r-\mu r-1}{r}-\log (L) \\
& -\log (\Gamma(\mu))+(\mu-1) \psi(\mu r-r+1) .
\end{aligned}
$$

From the above, the Shannon entropy represented in Eq. (47) can be finally rewritten as

$$
h(Z)=(1-\mu) \psi(\mu)+\log (\Gamma(\mu) \cdot L)+\mu,
$$

and the proof of the statement is completed.

Similarly to Rényi entropy case, for i.i.d. random variables, the Shannon entropy follows

$$
h(Z)=(1-m n) \psi(m n)+\log (\Gamma(m n) \cdot L)+m n .
$$

\section{NUMERICAL RESULTS}

Here, we provide numerical results of the analytical expressions obtained in section above. Specifically, for convenient positive constants $k_{1}$ and $k_{2}$, we evaluate the Rényi and Shannon entropies of a random variable $Z_{2}$ defined as

$$
Z_{2}=k_{1} R_{1}^{2}+k_{2} R_{2}^{2}
$$

where, $R_{1}$ and $R_{2}$ are independent Nakagami- $m$ distributed as follows

$$
R_{1} \sim \mathcal{N} \mathcal{K}_{m}(1.5,1) \text { and } R_{2} \sim \mathcal{N} \mathcal{K}_{m}(1,2) .
$$

Note that, $\mu=1.5+1=2.5$ and, based on Eq. (30), we obtain $k_{1}=1, k_{2}=3$ and $L=1.5$. Consequently, $Z_{2}$ is Gamma distributed as

$$
Z_{2} \sim \gamma(2.5,1.5)
$$

and given by

$$
Z_{2}=R_{1}^{2}+3 R_{2}^{2}
$$

Hence, using some numerical substitutions, the Rényi entropy of order $r$ in Eq. (41) reduces to

$$
\begin{aligned}
h_{r}\left(Z_{2}\right) & =\frac{1}{1-r}[(-1.5 r-1) \log (r)+0.405(1-r) \\
& +\log (\Gamma(1.5 r+1))-0.2847 r] \text { nats, }
\end{aligned}
$$


while the Shannon entropy of $Z_{2}$ is given by a simple numeric value obtained from Eq. (51). Precisely,

$$
h\left(Z_{2}\right)=2.1354 \text { nats. }
$$

Finally, in order to graphically illustrate such results, we plot in Fig. 1 Rényi and Shannon entropies for different values of $r$. Obviously, in Rényi entropy curve exists a point of discontinuity over $r=1$, which is compensated by Shannon entropy.

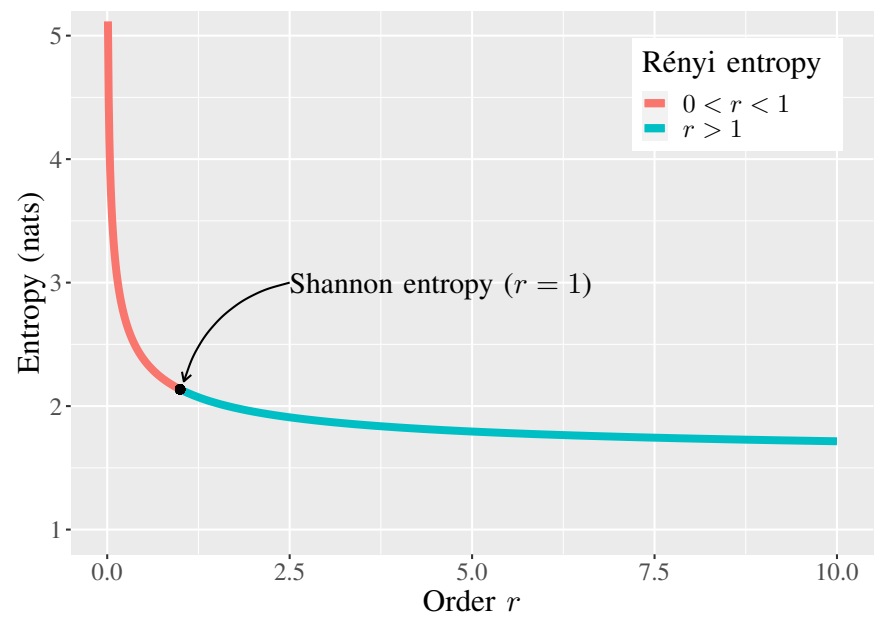

Fig. 1. The Rényi and Shannon entropies of $Z_{2}$ for different values of $r$.

\section{CONCLUSIONS}

In this paper, we derived closed-form expressions for Shannon and Rényi differential entropies over the weighted sum of squared Nakagami- $m$ random variables including the case of independent and identically distributed variables. From the proposed hypotheses, it was possible to transform such weighted sum into a simple Gamma distribution. In addition, after some algebraic manipulations, the respective entropies can be written in terms of special functions, specifically, gamma and digamma functions. Finally, we presented an example to illustrate the mathematical formulations developed in this work.

\section{REFERENCES}

[1] C. E. Shannon, "A mathematical theory of communication". Bell Syst. Tech. J., v. 27, pp. 379-423, 1948.

[2] T. M. Cover and J. A. Thomas, Elements of Information Theory (Wiley Series in Telecommunications and Signal Processing), WileyInterscience, 2006.

[3] R. R. Perera, T. S. Pollock and T. D. Abhayapala, "Bounds on mutua information of Rayleigh fading channels with Gaussian input", 2005 Australian Communications Theory Workshop, pp. 62-67, Feb. 2005.

[4] S. Cruces, R. Martín-Clemente and W. Samek, "Information Theory Applications in Signal Processing", Entropy, v. 21, n. 653, 2019.

[5] F. Escolano, P. Suau and B. Bonev, Information Theory in Computer Vision and Pattern Recognition. Springer Publishing Company, Incorporated, 2009.

[6] P. Bereziński, B. Jasiul and M. Szpyrka, "An Entropy-Based Network Anomaly Detection Method", Entropy, v. 17, n. 4, pp. 2367-2408, 2015.

[7] D. J. C. MacKay, Information Theory, Inference \& Learning Algorithms. Cambridge University Press, 2002.
[8] R. V. Belavkin, P. M. Pardalos, J. C. Principe and R. L. Stratonovich, "Theory of Information and its Value", Information theory and the second law of thermodynamics, pp. 391-408. Springer International Publishing, 2020.

[9] J. Yang, "INFORMATION THEORETIC APPROACHES IN ECONOMICS", Journal of Economic Surveys, v. 32, n. 3, pp. 940-960, 2018.

[10] M. M. Wilde, Quantum Information Theory. Cambridge University Press, 2013

[11] A. Rényi. "On measures of entropy and information", Proceedings of the fourth Berkeley symposium on mathematics, statistics and probability, v. 1, pp. 547-561, 1960.

[12] C. Tsallis, "Possible generalization of Boltzmann-Gibbs statistics", Journal of Statistical Physics, v. 52, pp. 479--487, 1988.

[13] F. Nielsen and R. Nock, "A closed-form expression for the Sharma-Mittal entropy of exponential families". Journal of Physics A: Mathematical and Theoretical, v. 45, n.3, Dec. 2011.

[14] M. Yacoub, J. Bautista, and L. G. de Rezende Guedes, "On Higher Order Statistics of the Nakagami-m Distribution". IEEE Trans. Veh. Technol., v. 48, n. 3, pp. 790_794, May 1999.

[15] I. Ansari, F. Yilmaz, M.-S. Alouini, and O. Kucur, "On the Sum of Gamma Random Variates with Application to the Performance of Maximal Ratio Combining over Nakagami-m Fading Channels", in 2012 IEEE 13th International Workshop on Signal Processing Advances in Wireless Communications (SPAWC), pp. 394--398, 2012.

[16] C. Zhong, K.-K. Wong, and S. Jin, "On the Ergodic Capacity of MIMO Nakagami-Fading Channels", in IEEE Int. Symp. on Inform. Theory (ISIT), pp. 131--135, Jul. 2008.

[17] I. Ansari, F. Yilmaz, M.-S. Alouini, and O. Kucur, "New results on the sum of Gamma random variates with application to the performance of wireless communication systems over Nakagami- $m$ fading channels", Transactions on Emerging Telecommunications Technologies, v.28, n. 1 , pp. e2912, 2017.

[18] A. A. P. Guimarães, M. Kountouris, C. C. Cavalcante, "Bounds and approximations on the ergodic mutual information of spatially correlated Nakagami-m MIMO channels", Transactions on Emerging Telecommunications Technologies, v. 27, n. 6, pp. 842-856, 2016.

[19] S. K. Yoo, P. C. Sofotasios, S. L. Cotton, S. Muhaidat, O. S. Badarneh and G. K. Karagiannidis, "Entropy and Energy Detection-Based Spectrum Sensing Over $\mathcal{F}$-Composite Fading Channels", IEEE Transactions on Communications, v. 67, n. 7, pp. 4641-4653, Jul. 2019.

[20] A. T. Asyhari, T. Koch, A. G.-i Fàbregas, "Nearest Neighbor Decoding and Pilot-Aided Channel Estimation for Fading Channels", Entropy, v. 22, n. 9, 2020.

[21] T. Xifilidis and K. E. Psannis, "Wireless fading channels performance based on Taylor expansion and compressed sensing: A comparative approach", International Journal of Communication Systems, v. 34, n. 8, pp. e4794, 2021.

[22] A. Papoulis and S. Pillai, Probability, Random Variables and Stochastic Processes. Fourth edition. McGraw Hill, 2002.

[23] L. Andrews, Special Functions of Mathematics for Engineers. Oxford science publications, 1998.

[24] W. R. Wade, An Introduction to Analysis. Pearson Education, 2014.

[25] I. S. Gradshteyn and I. M. Ryzhik, Table of Integrals, Series, and Products. Elsevier/Academic Press, 2007.

\section{APPENDIX}

Here, we proof that $\nu=\mu r-r+1$ is a positive number. By contradiction, suppose there is $r>0$, with $r \neq 1$, such that $\nu \leq 0$. Thus,

$$
\mu \leq 1-\frac{1}{r} .
$$

If $0<r<1$, then $0<1<1 / r$ and $\mu<0$, which contradicts the assumption that $\mu \geq 1$ (see Eq. (31)). In turn, if $r>1$, we obtain

$$
\mu \leq 1-\frac{1}{r}<1 \leq \mu
$$

i.e., $\mu<\mu$, which implies an another contradiction. Therefore, $\nu=\mu r-r+1>0$, for all $r>0$, with $r \neq 1$. 